\title{
Donner une voix aux Inuit urbains : «Photovoice» comme une pratique de multilittératie dans la construction d'identité et de savoirs transfrontaliers
}

\author{
Gabriele Budach \\ G.Budach@soton.ac.uk \\ UNIVERSITY OF SOUTHAMPTON \\ Donna Patrick \\ donna_patrick@carleton.ca \\ CARLETON UNIVERSITY
}

\begin{abstract}
Résumé
Cet article présente des résultats d'un projet de recherche ethnographique et collaboratif s'intéressant aux pratiques multimodales de littératie auprès de la communauté Inuit urbaine à Ottawa. Conçue comme un projet de recherche-action l'étude a été menée par des chercheurs universitaires en collaboration avec le programme de littératie familiale attaché au centre d'enfants Inuit à Ottawa (OICC). Cette recherche porte sur la méthode «Photovoice» et sa mise en pratique dans le contexte de la communauté Inuit urbaine. Notre recherche poursuit deux objectifs. Le premier consiste à explorer la façon dont "Photovoice » a été appropriée par la communauté Inuit urbaine comme un moyen de stimuler la réflexion sur l'expérience de migration transfrontalière et la création d'identités Inuit urbaines. Le deuxième cherche à reconnaître la méthode "Photovoice » comme une pratique sociale de littératie et à évaluer sa place comme méthodologie de recherche au sein des New Literacy Studies. Nos résultats de recherche suggérons que «Photovoice» en tant que méthode promouvant la multilittératie peut servir d'outil de réflexion et d'apprentissage dans le contexte communautaire des Inuits urbains ainsi que dans d'autres contextes.
\end{abstract}

Mots-clés : littératie, multilittératies, multimodalité, «Photovoice», Inuits urbains

\begin{abstract}
This paper reports on a collaborative, action-based research project exploring multimodal literacy practices among urban Inuit in Ottawa. Working in the context of the family literacy program of the Ottawa Inuit Children's centre, we examine two "Photovoice" activities conducted by Inuit
\end{abstract}

\section{(C)CAHIERS DE L'ILOB Vol. 2, 2011 35-55}


children, youth, and adults in 2009 and 2010. The goals of this examination are twofold: 1) to explore "Photovoice" as a multimodal literacy activity, and its appropriation by urban Inuit as a means to creatively explore, reflect upon, and represent aspects of urban Inuit identity and experiences; and 2) to situate "Photovoice" methodologically and theoretically in the realm of New Literacy Studies, which sees literacy as a social and cultural practice, rather than a set of discrete linguistic skills to be taught, learnt, and acquired. In this way, "Photovoice", drawing on the visual, oral, and scriptural modes, is seen as an ideal type of literacy activity in community-based educative contexts, such as urban Inuit in Ottawa, with wider application across contexts.

Key words: literacy, multiliteracies, multimodality, "Photovoice", urban Inuit

\section{Introduction}

Ce texte traite de la communauté Inuit en milieu urbain. Il s'inscrit dans le cadre d'une étude ethnographique plus large portant sur les pratiques de littératie au sein de cette communauté migratoire. Conçue comme projet de rechercheaction l'étude est basée sur la collaboration de chercheurs universitaires et d'une organisation communautaire Inuit, le centre d'enfants Inuit à Ottawa (OICC). Le présent texte se penche sur une activité précise explorant la méthode «Photovoice» et sa mise en pratique au sein de ce centre communautaire. Notre recherche poursuit deux objectifs. D'une part, elle vise à explorer la façon dont cette méthode a été appropriée par la communauté Inuit urbaine comme moyen de stimuler la réflexion sur l'expérience de migration transfrontalière et la création d'identités Inuit urbaines. D'autre part, notre étude cherche à reconnaître la méthode « Photovoice» comme une pratique sociale de littératie et à évaluer sa place en tant que méthodologie de recherche au sein des études sur la littératie et des New Literacy Studies.

La présente étude mise sur les processus d'identification sociale, culturelle et linguistique des Inuit urbains. Elle aide à mettre en lumière les positionnements individuels et collectifs d'une communauté transnationale distincte et de ses traits et besoins socio-culturels particuliers (Tomiak et Patrick, 2010). Au niveau théorique, cette recherche contribue à approfondir la compréhension de la multilittératie comme ensemble de pratiques socialement situées et différenciées. Plus particulièrement, l'étude souligne le caractère intégré de pratiques multimodales, langagières et visuelles. De plus, elle met en valeur l'importance particulière de l'espace comme un territoire multidimensionnel, naturel et institutionnel, et précise son rôle dans la construction de savoir et d'identité Inuit urbains. 


\section{Cadre conceptuel}

Notre recherche s'inscrit dans le courant d'études des New Literacy Studies (Street, 1995, 2000; Barton, 1994; Martin-Jones et Jones, 2000; Barton, Hamilton et Ivanč, 2000). Ce paradigme définit la littératie comme une pratique sociale historiquement et culturellement située et l'envisage comme un ensemble de pratiques multiples, possiblement multilingue et multimodale, dont la composition peut varier selon le contexte. Vu le caractère socialement contextualisé de toute pratique de littératie il paraît évident que des pratiques spécifiques peuvent prendre des formes potentiellement hétérogènes et certainement non généralisables. Une telle conception se trouve en fort contraste avec des définitions de littératie universalisantes qui la déterminent comme un ensemble de compétences mesurables (Barton, 1994). De telles approches imaginent l'acquisition de la littératie comme un processus d'apprentissage linéaire, plus ou moins unidirectionnel et monodimensionnel passant d'un niveau de compétence inférieur à un niveau supérieur. Cette définition vise à établir des résultats d'apprentissages d'un groupe précis afin de mesurer de façon non équivoque le progrès d'une population estudiantine donnée. L'imagination d'un tel parcours préconçoit non seulement une vision homogénéisante du processus d'apprentissage, il construit aussi l'apprenant débutant comme une personne immature, voire déficitaire.

En général, l'idéalisation de l'apprenant et l'imagination d'un parcours d'apprentissage précis constituent la base des modes d'évaluation qui sont employés surtout en milieu scolaire. Suite à l'implantation des politiques néolibérales en milieu éducatif depuis les années 1990, de telles pratiques d'évaluation ont gagné de l'importance également dans le contexte de l'éducation aux adultes (Budach, 2003). Pourtant, elles ont tendance à faire preuve d'un certain manque de sensibilité socioculturelle et linguistique en ignorant les parcours de socialisation diversifiés. En fait, ce ne sont que certains types de capital linguistique et de formes de savoirs que l'école valorise. Ce traitement inégal continue à être une source d'exclusion sociale. Le même constat peut être fait sur les façons de représenter le savoir en milieu scolaire qui, dans le passé, avaient tendance à privilégier les modes langagiers.

La présente recherche s'inscrit dans une optique alternative telle que proposée par les études sur la multiliteracy (Cope et Kalantis, 2000). Cette approche considère tout apprentissage comme un acte de création de sens possédant, en fait, un caractère multimodal (Kress, 2001). Une telle conception prend en considération le fait que l'objet d'apprentissage peut être construit et représenté sous différentes formes, en mode langagier, ou bien dans d'autres modes extra-linguistiques, visuels ou gestuels. En nous basant sur nos résultats de recherche, nous tenons à souligner l'importance d'une variété de modes qui peut stimuler l'apprentissage de manière importante. Dans cette 
étude, nous explorons comment la création d'artefacts et la circulation d'objets créés peuvent contribuer à l'apprentissage interactif au sein de la communauté Inuit urbaine.

Nous postulons que la méthode « Photovoice» est un outil approprié et apte à faire fructifier l'apprentissage multimodal. Choisissant comme point de départ la photographie, elle privilégie d'abord le mode visuel. Ceci n'exclut pas pour autant des opportunités variées de mener des activités de littératie en mode langagier, oral ou écrit. La méthode propose un format ouvert et flexible. Elle invite au traitement de thèmes divers et permet la création d'artefacts individuels dont la signification peut être discutée collectivement. Ainsi, un espace de communication se crée qui peut aider à forger un sentiment identitaire, un sens de la collectivité, et une expérience d'inclusion sociale. Cette activité qui est perçue par la communauté comme étant très positive peut aussi contribuer à changer l'image de la littératie comme plus inclusive et valorisant des activités communautaires au même titre que celles menées en contexte scolaire. Cette dimension est importante, car souvent les pratiques de littératie de communautés minoritaires ne sont pas prises en compte de façon adéquate par l'école.

\section{L'approche méthodologique «Photovoice»}

La méthodologie de «Photovoice» a été développée d'abord en sciences sociales dans le domaine des études de la santé (Wang et Burris, 1997). S'inspirant des études féministes et de la philosophie du développement communautaire de Paulo Freire (1973) la méthode «Photovoice» a visé, pour la plupart, des communautés minoritaires ou marginalisées, $78 \%$ de la totalité des études étant menées avec la participation et en collaboration avec des femmes (Willson, Green, Haworth-Brockman et Rapaport Beck, 2006).

La méthode devait permettre aux membres des communautés ciblées de mieux saisir leur propre condition et de contribuer à la changer activement (Wang et Burris, 1997; Wang, Yi, Tao et Carovano, 1998). «Photovoice» a été conçu comme un processus qui par l'intermédiaire d'une technique photographique aide à forger des identités et des représentations d'individus et de communautés (Wang, Cash et Powers, 2000). La méthode telle qu'envisagée par ces chercheurs visait trois objectifs :

- permettre aux communautés de saisir leur forces et défis et de réfléchir à des stratégies pour entraîner du changement;

- promouvoir le dialogue critique et la construction de savoir au sujet de thèmes clés, par le moyen d'images photographiques qui devraient être discutées en grand ou petits groupes; et

- attirer l'attention des représentants politiques. (Catalani et Minkler, 2010, p. 369) 
À l'heure actuelle, l'approche "Photovoice" jouit d'une grande popularité qui se reflète dans un nombre croissant d'études dans des domaines diversifiés. Les premières recherches de ce type ont été menées avec des femmes dans un village de la province Yunnan en Chine portant sur des questions de santé et de justice sociale (Wang, 1999; Wang et Burris, 1994, 1997). Dans des études plus récentes le répertoire de questions de recherche s'est élargi considérablement. Celles-ci incluent les conséquences médicales et sociales causées par la violence (Lykes, Blanche et Hamber, 2003) ainsi que la discrimination raciale et sexuelle (Graziano, 2004). L'éventail d'études couvre différentes générations et milieux institutionnels, étant orientés vers des jeunes (Wilson et al., 2007), des retraités (Baker et Wang, 2006), des programmes situés en contexte scolaire ou communautaire (Wilson et al., 2007) ainsi qu'en milieu de travail (Gallo, 2002). Les études inspirées par la méthodologie «Photovoice» englobent plusieurs continents, incluant l'Asie, l'Afrique, l'Amérique du Nord, l'Amérique Latine et l'Europe, et touchent également à une variété de groupes sociaux et de catégories sociales, comme la race, l'ethnicité et le sexe.

Suivant les objectifs de recherche en sciences sociales, la plupart des études menées jusqu'ici n'a mis que relativement peu en valeur les aspects éducatifs ou reliés à la littératie. Pourtant, cet angle est prometteur et mérite d'être exploré davantage. Afin d'illustrer ce potentiel, nous allons citer deux études à titre d'exemple qui éclairent les liens entre les aspects de littératie et son rôle au sein d'un milieu social spécifique.

La première étude menée par Melina L. Gallo (2002) est située en milieu de travail. Ici, la méthodologie de «Photovoice» a été employée comme approche alternative dans l'enseignement de travailleurs d'origine migratoire (venant de Colombie, du Mexique, du Vietnam et de l'ex-Yougoslavie) en remplaçant des cours traditionnels d'anglais langue seconde. L'étude a montré comment à travers la mise en pratique de «Photovoice» qui a remplacé les cours d'anglais traditionnel les travailleurs d'usine ont pu découvrir une forme de littératie nouvelle et très significative pour eux. "Photovoice» a offert un moyen qui leur permettait de mieux comprendre leur environnement de travail, les processus de production (de chaîne) et la structuration des relations du pouvoir au sein de l'usine. L'auteur note que les cours d'anglais tels qu'offerts traditionnellement ont été conçus par des professionnels de service de langue et en collaboration avec les chefs d'entreprises mais sans consultation avec la population d'apprenants. En conséquence, ces cours ignoraient les vrais besoins des apprenants et suffisaient d'abord aux demandes du personnel de gestion de l'entreprise pour lequel les cours de littératie avaient surtout une fonction symbolique. La littératie est généralement vue comme une compétence importante dans le milieu de travail, mais les contenus offerts par les cours étaient, en fait, très détachés des réalités et besoins de l'environnement de travail concret 
en question. Misant d'abord sur l'enseignement du lexique et des structures grammaticales, les cours n'apportaient que peu à l'intégration des travailleurs dans le processus de travail et rien à leur avancement en termes de carrière professionnelle (Gallo, 2002, p. 52).

L'introduction de la méthode «Photovoice» a permis aux travailleurs de réfléchir à leur condition de travail et d'initier un échange avec d'autres collègues de travail par l'intermédiaire d'artefacts (des photos et des commentaires écrits) qui étaient affichés au panneau d'information de l'usine. De cette manière, la méthode «Photovoice » a fait découvrir aux travailleurs une littératie très différente de celle associée aux cours d'anglais habituels. En plus, l'apprentissage a non seulement été efficace au niveau de l'apprenant individuel, mais il a également généré un impact sur le milieu du travail plus globalement, amenant des changements, par exemple par rapport aux politiques de communication et au régime de nettoyage au sein de l'entreprise.

La deuxième étude que nous citons ici a été menée par Strack, Magill et McDonagh (2004) dans un «programme après-l'école» pour des adolescents issus des milieux socialement défavorisés au Sud-Est de Baltimore. Ce projet réunissait 14 participants, âgés de 11 à 17 ans, dont le profil démographique reflétait celui du quartier, incluant des noirs non-hispaniques $(50 \%)$, blancs nonhispaniques (29\%), blancs hispaniques (7\%) et de race mixte (14\%). Les jeunes se sont réunis deux fois par semaine pendant une durée totale de 12 semaines. Dans un milieu social marqué par un taux de revenu très bas et la présence de drogues, d'abus sexuel et de violence, le projet avait pour but d'engager les jeunes adolescents dans une activité intéressante, exigeante et satisfaisante qui leur permettrait de réfléchir à leur identité, leur environnement (le quartier), mais aussi à leurs propres plans et projets de vie. Dans ce contexte, l'approche «Photovoice» devait aider les jeunes à développer une identité de soi positive, une responsabilité pour l'autre et des compétences sociales (Strack, Magill et McDonagh, 2004, p. 50). Équipés d'un appareil photo, les jeunes ont été encouragés à explorer leur quartier, à prendre des photos et à les discuter avec les autres participants au programme. Même si le projet comprenait d'autres éléments tels que l'écriture de textes accompagnant les photos, il n'était pas conçu ou considéré comme une activité de littératie.

Les deux études mentionnées plus haut mettent en valeur l'exploration d'un espace particulier, celui du milieu de travail (Gallo, 2002) ou bien celui du quartier (Strack et al., 2004). Ensuite, elles décrivent le développement d'un dialogue engagé et critique par rapport à cet environnement. Dans notre étude, nous partageons quelques-uns des points focaux des études mentionnées plus haut, tels que l'intérêt pour l'environnement et le dialogue entre les participants. Pourtant, les résultats de notre recherche suggèrent que dans le contexte de la communauté Inuit urbaine ces deux dimensions sont interprétées d'une 
manière particulière. Nous soutenons l'hypothèse que cette spécificité est dûe aux besoins spécifiques de cette communauté transnationale en situation de migration dont les membres cherchent à connecter deux mondes et modes de vie différents, celui de la ville et celui de l'Arctique.

En contraste avec d'autres études, les participants de notre recherche explorent l'espace d'une façon particulière. Ils le découvrent comme une zone à dimensions multiples dans laquelle différents milieux de vie et traditions culturelles se croisent. L'environnement, tel que représenté par la photographie, devient un espace de traduction et de transmission de savoir culturel qui se reconstitue à travers le dialogue intergénérationnel entre membres de la communauté Inuit urbaine. Dans cette situation le dialogue devient un moyen privilégié afin de créer des liens intergénérationnels. De plus, l'élément dialogique valorise le mode oral qui est important traditionnellement dans la construction et la transmission du savoir chez les Inuits.

\section{Contexte de la recherche}

Le projet «Photovoice» fait partie d'un projet de recherche ethnographique plus large, intitulé «Littéracies multiples dans une communauté Inuit urbaine » (Multiliteracies in an urban Inuit community) qui est financé par le CRSH (période de financement : 2008-2011). Le projet est mené en collaboration avec le Centre de littératie familiale (Family Literacy Centre) qui est un programme spécifique au sein du Centre d'enfants Inuit à Ottawa (Ottawa Inuit Children Centre, OICC) à Vanier. La collaboration avec la communauté Inuit à Ottawa et le travail ethnographique au sein de cette communauté ont été initiés par Donna Patrick en 2004. Présentement, notre partenaire de recherche privilégié est le Centre d'enfants Inuit à Ottawa qui a été fondé en 2005. Le centre fournit des services aux familles et leurs enfants Inuit couvrant différentes tranches d'âge dès la naissance jusqu'à l'âge de 13 ans. Il organise également le programme « Head Start » desservant des enfants à l'age préscolaire.

Les Inuits vivant en contexte de ville peuvent être considérés comme une communauté trans-nationale (Tomiak et Patrick, 2010). Originaires de vastes territoires dans le Nord, ils proviennent de communautés géographiquement distantes et socio-linguistiquement diversifiées. Le territoire des communautés Inuit comprend les quatre régions suivantes : Nunavut, Nunavik (le Grand Nord québécois), Nunasiavut (le Labrador) et Inuvialuit (l'Arctique de l'Ouest), couvrant environ $40 \%$ du territoire du Canada. Ces territoires ont été établis suite au processus de revendication territoriale entre 1975-2005 et sont unifiés de nos jours sous le nom de «Inuit Nunaat». Au total, il y a 53 communautés qui sont éparpillées à travers l'Arctique. Le paysage linguistique est également très diversifié, c'est pourquoi il est maintenant admis d'utiliser le terme «les langues Inuit » au pluriel pour référer à l'ensemble des variétés d'inuktitut. 
La communauté Inuit à Ottawa est la plus grande vivant en dehors de 1'Arctique. Le nombre estimé de cette population varie selon les sources entre 800 et 1500 . Quant à l'origine de cette population, les résultats d'une enquête menée par le OICC dans la région d'Ottawa (OICC Nipivut Report, 2009) a révélé que $70 \%$ des familles sont originaire de Nunavut, $15 \%$ de Nunasiavut et 5\% de Nunavik et Inuvialuit. Ceci entraîne également l'hétérogénéité de la communauté Inuit urbaine d'un point de vue linguistique puisque différentes variétés de langues Inuit sont parlées dans ces régions de l'Arctique.

La communauté Inuit urbaine à Ottawa est hétérogène aussi par rapport à d'autres aspects. Ceux-ci concernent le revenu, le quartier de résidence en ville, l'usage des langues à la maison et la durée du séjour à Ottawa. Environ $40 \%$ des familles utilisent l'anglais et l'inuktitut à la maison, $40 \%$ de la population Inuit vivent en ville depuis plus de 10 ans, et $25 \%$ entre 7 et 10 ans. Quant à la motivation de la migration, la majorité des gens indiquent des raisons liées au travail, à la famille, à l'éducation et à la santé.

\section{La méthodologie «Photovoice» en contexte Inuit urbain}

Deux projets « Photovoice» ont été organisés jusqu'ici au sein du centre communautaire OICC à Ottawa. Le premier eut lieu en mars 2009, le deuxième a été organisé en avril 2010. Le premier projet faisait partie d'un programme de recherche plus ample, soutenue par l'initiative gouvernementale (Urban Aboriginal Strategy) et comprenant deux éléments ; une enquête statistique auprès de la communauté Inuit urbaine et le projet «Photovoice». Les deux activités avaient pour but d'évaluer les besoins de la communauté Inuit à Ottawa et de contribuer à l'amélioration des services communautaires au sein de cette communauté (OICC Nipivut Final Report, 2009, p. 8). Le premier élément, l'enquête, a recueilli des renseignements auprès d'environ 80 parents d'enfants (entre 0 et 18 ans) grandissant à Ottawa afin d'établir un portrait intergénérationnel de la communauté. La deuxième activité, un projet suivant la méthodologie de «Photovoice », a attiré l'intérêt de 17 participants, 9 adultes et 8 enfants (entre 4 et 14 ans). Quant au profil linguistique, le répertoire langagier des participants était varié. Tous parlaient l'anglais comme langue majoritaire de l'environnement. Les personnes ayant grandi dans l'Arctique et nouvellement arrivées du Nord tendent à être bilingues et maîtrisent l'anglais et une ou plusieurs variétés de langues inuktitut, tandis que ceux et celles qui ont grandi en ville possèdent souvent l'anglais comme langue dominante et disposent de connaissances restreintes en inuktitut. Pourtant, tous partagent un intérêt pour la langue et la culture inuktitut, et certains d'entre eux, surtout parmi les enfants, l'apprennent activement au sein des programmes de l'OICC. Le centre offre quotidiennement de l'instruction en inuktitut pour des enfants (de 2 à 5 ans) dans le programme «Head Start», et des cours de langue en inuktitut pour 
adultes une fois par semaine.

Grâce au succès du premier projet «Photovoice», qui était fortement ressenti par la communauté Inuit locale, un deuxième projet a été organisé en avril 2010. Cette deuxième édition a attiré l'intérêt de 8 adultes et 7 enfants. La visée de ce deuxième projet était quelque peu différente par rapport à celle du premier, surtout quant à l'ancrage scientifique et au support institutionnel. Le premier projet mettait plutôt l'accent sur l'évaluation des besoins sociomédicaux de la communauté. Par contre, le deuxième s'intéressait davantage aux pratiques de littératie telles qu'elles ont émergé au sein d'une communauté migratoire multilingue. La deuxième édition a été organisée par le programme de littératie familiale du OICC et une équipe de l'université Carleton sous la direction de Donna Patrick. Le support financier provenait de fonds de recherche d'un projet du CRSH qui couvre aussi une partie des coûts de personnel du programme de littératie au sein du OICC.

Par la suite, nous décrivons quelques caractéristiques des deux projets «Photovoice» qui ont été menés au sein du centre OICC. Nous mettons en parallèle les deux éditions (2009 et 2010) et les comparons à la conception de l'approche «Photovoice». Nous mettons également en évidence des points de comparaison avec d'autres projets « Photovoice» menés dans des contextes différents.

Semblables à d'autres projets adoptant une méthodologie «Photovoice », les deux projets offraient une série de trois ateliers sur une durée de quatre semaines. Chaque atelier était consacré à des activités précises.

Le premier atelier s'ouvrait sur une introduction aux objectifs et à la méthodologie de l'approche «Photovoice». Ensuite, une présentation des aspects techniques de la photographie était faite par des photographes professionnels, en tenant compte de l'hétérogénéité du groupe de participants, qui comprenait aussi bien des personnes ayant des connaissances approfondies en photographie que des débutants pour lesquels l'atelier constituait la première expérience dans ce domaine. Suite à la présentation par les experts les participants à l'atelier ont été invités à discuter en groupe des photos qui étaient mises à leur disposition afin de mettre en pratique leur savoir nouvellement acquis. Le deuxième atelier qui a eu lieu deux semaines plus tard comprenait une discussion des photos prises par les participants dans leur entourage et le choix des deux meilleures photos suite à des échanges et discussions critiques entre les participants. Pendant le troisième atelier, mené la semaine suivante, les participants ont présenté leurs photos devant tout le groupe et ont commencé la conception et rédaction de textes qui devaient accompagner ces photos.

Comme dans d'autres projets du type «Photovoice» les artefacts finaux ont été encadrés et assemblés dans une exposition. Cette collection a été montrée dans différents espaces de la ville d'Ottawa, présentant l'image d'une com- 
munauté Inuit urbaine peu connue du grand public. Les sites publics où l'exposition a été présentée incluaient des cafés, une maison de services de santé, l'entrée d'un bâtiment gouvernemental et la faculté de médecine de l'université. Ce «voyage » a permis de non seulement transcender des frontières spatiales, mais aussi de rejoindre des publics variés appartenant à des milieux professionnels et classes sociales divers.

Le choix du format (l'exposition) pour partager les résultats du projet est une pratique courante au sein de «Photovoice» et peut être considéré comme une caractéristique essentielle de cette méthode. Cependant, la collection Inuit urbaine comporte des caractéristiques spécifiques unissant des artefacts multimodaux bilingues. Tous les textes accompagnant les photos ont été écrits par les participants en anglais, la langue dominante de l'environnement. Par la suite, ces textes ont été traduits de l'anglais en langue inuktitut par une personne maîtrisant l'inuktitut couramment à l'oral et à l'écrit. Cet ajout a mené à la transformation des artefacts en textes multimodaux bilingues et à la présentation des Inuit urbains comme une communauté bilingue et linguistiquement distincte.

Cet acte est intéressant à plusieurs niveaux. Premièrement, il crée une voix bilingue « unifiée », en anglais et inuktitut, car la traduction a été faite par une seule et même personne de la région arctique de Baffin. Ce choix a déterminé la sélection d'une variété de langue Inuit particulière, ce qui a entraîné l'homogénéisation linguistique du corpus entier. Deuxièmement, cette homogénéisation a mené à une certaine neutralisation des répertoires linguistiques de participants individuels puisque certains d'entre eux n'écrivent pas l'inuktitut ou bien choisiraient d'écrire dans une autre variété ou langue Inuit.

Enfin, nous voulons souligner un dernier aspect quant au cadre conceptuel plus large qui marque une différence entre le premier et le deuxième projet. Inscrite dans un programme de recherche en sciences sociales, la première édition de « Photovoice » mettait l'accent sur la conscientisation sociale et la sensibilisation aux problèmes au sein d'une communauté qui est perçue souvent comme socialement marginalisée et en manque de ressources. Deux questions ont été proposés par les organisateurs afin de guider la réflexion des participants :

1. Qu'est-ce qui vous plaît dans votre communauté à Ottawa?

2. Qu'est-ce que vous aimeriez changer?

Ces questions cherchaient à inviter les participants à réfléchir de façon critique aux conditions de leur vie en communauté et à imaginer des voies de changement.

Le deuxième projet, par contre, envisageait l'approche de «Photovoice » comme une activité de littératie. Ainsi, les participants étaient invités à découvrir leur environnement d'une manière nouvelle. En même temps, «Pho- 
tovoice » devait leur permettre de découvrir la littératie comme un concept et un ensemble de pratiques qui leur seraient utiles afin de mieux comprendre leur expérience migratoire et développer un sens de soi et de la communauté. Les questions proposées par les organisateurs de «Photovoice II » étaient les suivantes :

1. Où est-ce que tu te sens bien à Ottawa?

2. Où est-ce que tu te sens chez toi à Ottawa?

3. Où est-ce que tu te sens Inuit ou connecté à la culture Inuit à Ottawa?

\section{L'analyse du projet «Photovoice« comme un processus d'apprentis- sage multidimensionnel}

Nous nous pencherons ici sur l'analyse de données ethnographiques concrètes. Nous aborderons deux études de cas dont les données ont été recueillies lors de «Photovoice I et II ». Ces deux cas nous servent d'exemples pour mettre en lumière différentes façons dont les participants Inuit explorent l'espace. Ils nous aident à mieux comprendre comment les Inuit en milieu urbain :

1. construisent des identités et savoirs Inuit dans un contexte de migration transnationale,

2. créent des liens entre des pratiques et savoirs de littératie de types différents, reliant les contextes communautaire et scolaire.

La première étude de cas nous montre comment l'approche de «Photovoice » peut stimuler l'exploration de l'espace, réel et virtuel, afin de comprendre différents environnements, celui de la ville et celui de l'Arctique. Elle souligne également l'importance de la communauté Inuit et de ses institutions qui jouent un rôle primordial et indispensable dans la construction du savoir et de l'identité Inuit urbains. Dans cet exemple, nous assistons à la constitution de récits multiples qui se créent à base d'artefacts multimodaux et à travers le dialogue intergénérationnel. Ce dialogue met en rapport des membres de la communauté avec différents niveaux d'expérience, les plus expérimentés guidant l'apprentissage des plus jeunes.

La deuxième étude de cas nous montre comment l'exploration de l'espace naturel et de ses ressources matérielles, qui sont valorisés dans la tradition Inuit, peuvent stimuler l'apprentissage de la littératie. Cet exemple nous rappelle que l'acquisition du code alphabétique est une activité qui englobe différents mondes d'expérience de l'enfant et qui ne se limite ni à l'instruction formelle ni aux frontières spatiales des pratiques en milieu scolaire. 


\section{Étude de cas I : La co-construction collaborative de l'histoire de Clayton}

Les protagonistes de cette étude de cas sont Louisa et son fils, Clayton ${ }^{1}$, âgé de 5 ans au moment de notre collecte de données. La mère travaille pour le OICC à la direction du programme «Head Start», qui offre des activités de sensibilisation à la culture et aux langues Inuits pour des enfants à l'âge préscolaire. Son fils fréquente ce programme où il jouit d'un accès privilégié à des ressources culturelles et linguistiques de la communauté Inuit en milieu urbain.

Lors du projet «Photovoice II », auquel les deux participent, mère et fils travaillent ensemble. Par la suite nous retraçons quelques moments clés de cette collaboration intergénérationnelle. Notre analyse se base sur différentes sources de données; des photos prises par Clayton, des transcriptions d'interactions verbales, enregistrées ou notées lors des ateliers, et les textes accompagnant les photos tels qu'ils apparaissent dans l'artefact final.

Le premier moment que nous abordons dans notre analyse a été observé pendant le troisième atelier. Clayton présente ses deux photos sélectionnées au groupe comprenant tous les participants. Elles représentent des scènes de la vie Inuit dans l'environnement de l'Arctique que Clayton a créées à base de figures et de matériel mis à disposition par le programme «Head Start». La scène sur la première photo montre un igloo, un ours polaire [nanuk] et un chasseur Inuit. Sur la deuxième photo nous voyons un paysage similaire, mais l'ours polaire est plus présent et domine la partie droite de la photo (voir la Fig. 1).

Dans la présentation devant le groupe, Clayton assume le rôle du protagoniste qui explique ses photos au moyen d'histoires fictives. Par rapport à la première photo, il raconte (C : Clayton; I : chercheure) :

C : this is a hunter who is going for a seal. The nanuk [ours polaire] is watching on the ice

Par rapport à la deuxième photo il raconte l'histoire suivante :

C : and that one the guy is going for a seal and the other guy is sleeping and the nanuk is coming round the iglu

I : oh oh is he going to get him?

C : no

Dans cet extrait Clayton insuffle de la vie aux objets et aux personnes que l'on découvre sur la photo lorsqu'il imagine l'histoire d'une chasse dans le contexte arctique. Il représente des caractéristiques environnementales essentielles de la vie dans l'Arctique, comme la glace, l'igloo et l'ours polaire, et construit son histoire autour d'une activité clé de la vie Inuit, celle d'aller à

\footnotetext{
${ }^{1}$ Les noms des participants à notre recherche ont été changés afin de garantir leur anonymat.
} 


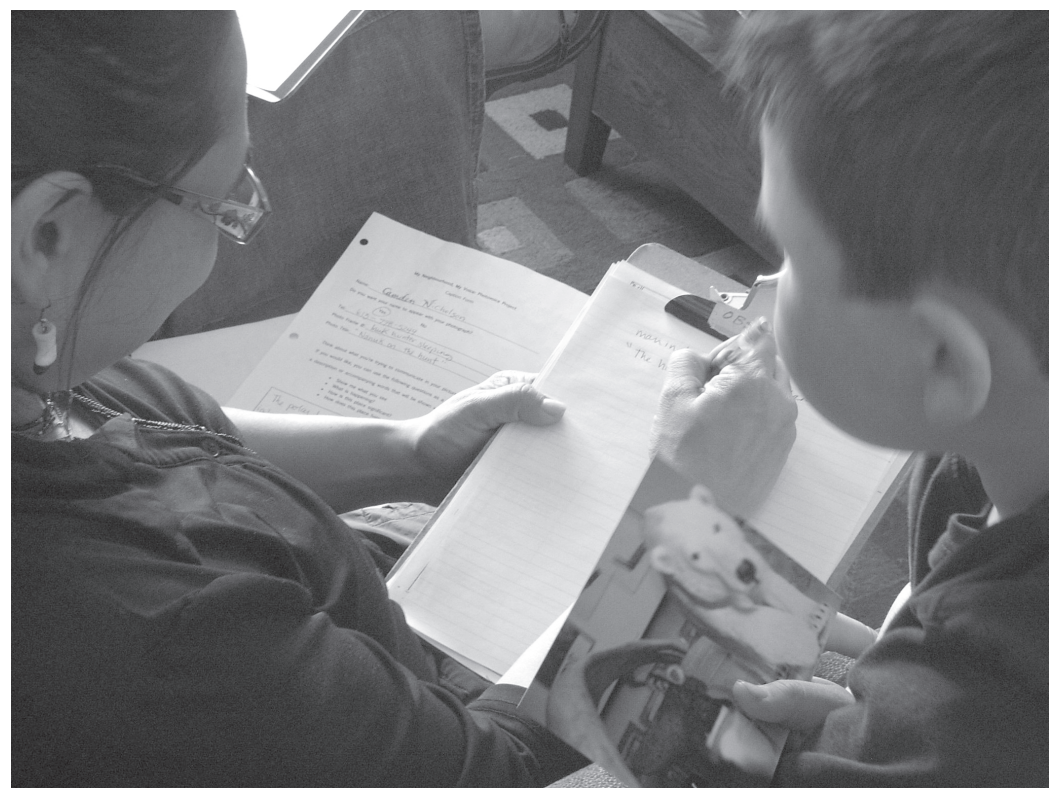

FIGURE 1

Clayton et Louisa

la chasse. Il fait preuve de son savoir culturel et linguistique et sait appeler l'ours polaire par son nom en inuktitut «nanuk». L'extrait rend compte d'un autre type d'interaction qui contribue à faire avancer la construction de l'histoire de Clayton. La chercheure, demandant à Clayton si l'ours polaire allait réussir à attraper le chasseur, pousse notre auteur à imaginer la poursuite du fil de l'histoire.

Plus tard pendant le même atelier nous sommes témoin d'une autre interaction mettant en lumière l'intégration du code écrit. La photo ci-dessus montre Clayton et sa mère qui collaborent à rédiger l'histoire. La photo montre Clayton, le constructeur des scènes photographiées en train de dicter son histoire à sa mère. Etant trop jeune pour l'écrire lui-même, il s'appuie sur l'aide de sa mère qui rédige l'histoire pour lui en anglais. Même si Clayton apprend l'inuktitut dans le programme «Head Start», il n'a pas encore de connaissances suffisantes pour imaginer une histoire dans cette langue. Sa mère qui a grandi en ville comme lui a été élevée dans un mariage mixte en langue anglaise puisque son père d'origine Inuit avait décidé de ne pas transmettre l'inuktitut à ses enfants.

Dans la composition de l'histoire le rôle de la mère ne se limite pas à 
copier les propos de l'enfant. Elle le guide en poussant le développement du récit par des questions comme «Qu'est-ce qui se passe ensuite?» ou «Qu'estce qui arrive au kayak? », ce qui aide l'enfant à construire son histoire. Le texte final de sa première histoire se lit ainsi :

This is me [Clayton] hunting for seals, I am in a kayak. The nanuk is hunting, he is hunting seals too. I see a seal and the nanuk didn't even see it. When I am close to home I will get out of my kayak. I took this picture at Head Start, where I learned about seal hunting.

Dans cette histoire Clayton apparaît dans des rôles multiples et agit comme conteur, personnage et acteur principal. Il adopte l'identité du chasseur Inuit et sait manœuvrer un kayak. Il entre même en compétition avec l'ours polaire et met à l'épreuve les capacités de cet animal qui est généralement reconnu comme un excellent chasseur. Il rejoint sa maison en kayak et imagine «être chez lui » dans un environnement arctique. Pour lui, comme enfant grandissant en ville, ce voyage fictif et l'imagination d'un monde si distant de sa réalité quotidienne représentent un moment d'identification important. À travers la création du récit fictif il découvre et expérimente un contexte de vie qui est loin des pratiques culturelles urbaines, mais qui l'aide à maintenir le contact avec la culture de sa famille, sa communauté et son héritage culturel Inuit. L'histoire de la chasse dans sa forme écrite est suivie d'un commentaire non-fictif qui illumine les conditions de cette création fictive. Le commentaire mentionne le programme «Head Start» et met en évidence l'importance du centre communautaire comme cadre référentiel et lieu d'apprentissage pour l'enfant. Le texte qui accompagne la deuxième photo est structuré de manière semblable. Il commence par une histoire fictive et se termine par des renseignements contextuels par rapport au programme «Head Start». Ce deuxième texte se lit :

Nanuk on the hunt :

The polar bear is creeping up on the igloo where one guy is sleeping and one is keeping guard. The guard is looking out for the nanuk, and will keep his little brother safe. I made this scene at Sivummut Head Start on the cultural table. I like head start because it has lots of Inuit stuff, and I started coming here when I was 2 years old.

Dans cet extrait le programme «Head Start» est appelé par son nom officiel «Sivummut Head Start», sivummut signifiant «apprendre ensemble » en inuktitut. Cette citation souligne le caractère socioculturel particulier du centre et identifie le $\mathrm{OICC}^{2}$ comme un lieu clé d'apprentissage et de formation identitaire pour la communauté Inuit urbaine. C'est au sein de cet espace

\footnotetext{
${ }^{2}$ La mission du programme se lit ainsi : "The mission of the Sivummut Head Start Program is to provide each child and family with a supportive learning environment that promotes Inuit culture and language.” Consulté le 29 août 2010, www.ottawainuitchildrens. com/eng/index.php?option=com_content $\backslash \&$ view=article $\backslash \&$ id $=36 \backslash \&$ Itemid $=41$.
} 
institutionnel que Clayton acquiert des connaissances sur les traditions et pratiques culturelles Inuit, telles que la chasse aux phoques qu'il met en pratique dans son récit fictif. On nous présente également la «table culturelle », endroit précis où des objets avec une signification culturelle Inuit sont mis à la disposition des enfants en tant que ressources de jeu et d'apprentissage : une peau de phoque, des vêtements portés en Arctique, des ustensiles nécessaires pour vivre et pour aller à la chasse ainsi que des reproductions de personnages ou d'objets en miniature comme ceux représentés sur les photos prises par Clayton. En tant que ressources de littératie ces objets forment le centre d'attention d'activités ludiques variées et le point de départ d'activités langagières telles que la récitation d'histoires en inuktitut ou de tâches liées à l'acquisition de savoirs à propos d'animaux, leurs noms en inuktitut, leurs caractéristiques, leur mode de vie et leur utilité pour la communauté Inuit vivant en Arctique.

«Head Start» est apprécié par la communauté Inuit urbaine comme un espace social et culturel très riche qui offre des ressources et un milieu propices à l'apprentissage. C'est dans cet espace que des enfants comme Clayton s'épanouissent et développent une familiarité avec la culture Inuit dès leur petite enfance. Cette identification positive est partagée également par la mère de Clayton, Louisa, qui exprime son rapport à cette institution dans une entrevue rétrospective menée après la fin du projet :

You know, really when it came down to it, both my son and I realize that Head

Start is like our second home

Ces propos confirment l'attachement de la mère et du fils à cet espace que la communauté Inuit urbaine a réussi à se créer à Ottawa. Dans le même entretien la mère souligne que le projet «Photovoice» lui paraissait un moyen adéquat qui répondait bien à ses propres besoins et à ceux de la communauté. Selon Louisa «Photovoice» serait susceptible de renforcer les relations sociales entre les membres de la communauté Inuit ainsi que d'encourager le dialogue intergénérationnel qui favoriserait également la transmission de savoir culturel Inuit :

And really, what the driving force for me to sign up was [to have] an activity to do with my son . . uh ... [...] Yeah, he still remembers the, you know, taking the pictures, and what the pictures meant and trying to come up with stories behind them. Like, just to me it was a really awesome bonding moment for us.

À la question de savoir si Louisa était intéressée à participer à une prochaine édition de «Photovoice», elle répond avec beaucoup d'enthousiasme :

MOST definitely. And I think this time I'd like to try it with my older son, who wasn't interested this time, but I think once this piece comes together and he sees my photos and then he sees his brother's photos, I think he will be much more inspired and want to try it and I would love to do it with him, because I think that, you know, his perspective would be really great ...] 


\section{Étude de cas II : Construire la lettre M}

La deuxième étude de cas présente l'exploration de l'espace sous une forme différente. Le protagoniste est Mike qui a 8 ans au moment de sa participation au projet «Photovoice I ». Notre analyse se penche sur des données visuelles et des activités captées dans la photo 2 (voir la Fig. 2) et la photo 3 (voir la Fig. 3).

La photo 2 montre l'enfant en train de composer son motif, qu'il prendra en photo quelque temps plus tard.

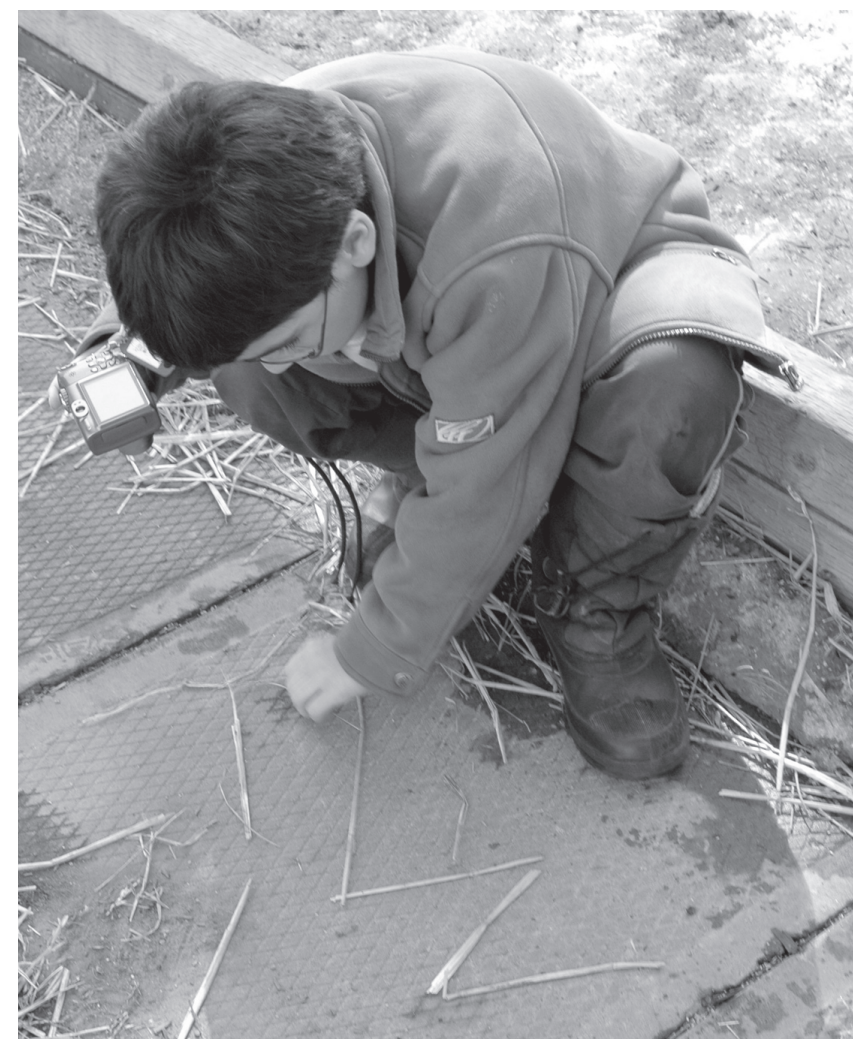

FIGURE 2

Mike construit la lettre M

La photo 3 montre l'artefact final tel qu'exposé à la fin du projet. Elle contient l'image photographiée, l'identification de son créateur et un texte accompagnant la photo écrit par Mike et traduit en inuktitut par une autre per- 


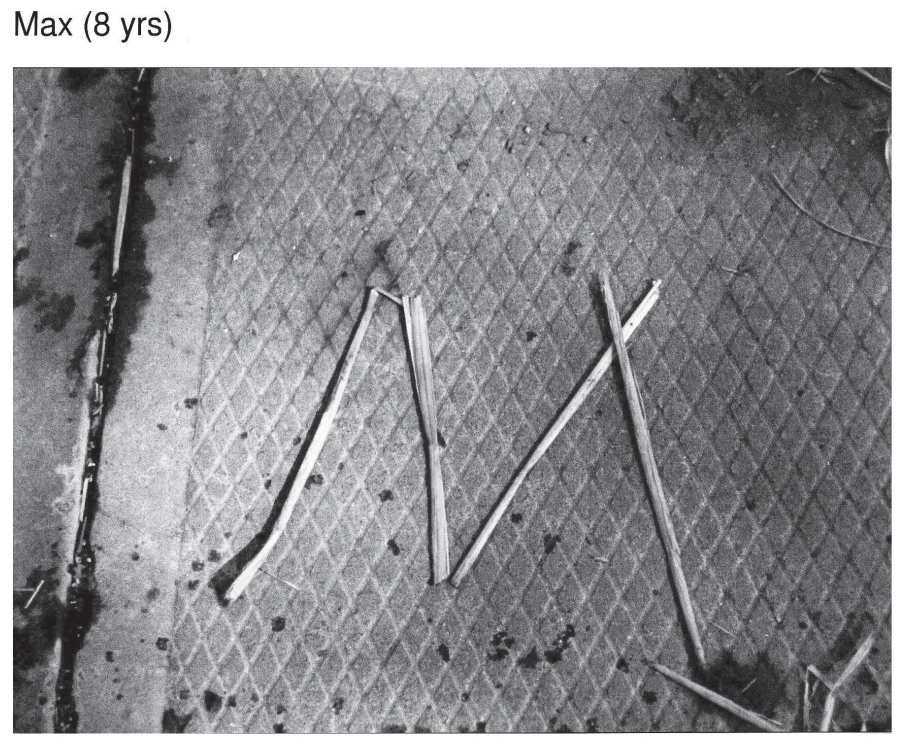

I like the letter "M" and I'm going to make a cloud of things that start with "M".

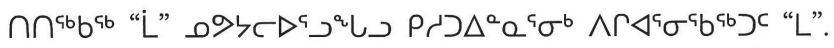

FIGURE 3

Mike, artefact final

sonne. Cette image donne une idée du format et de la composition d'autres artefacts dont une vingtaine du même type étaient inclus dans l'exposition.

Sur la photo 2 nous voyons l'enfant en train d'explorer l'espace. Cette activité se tient dans la cour du centre communautaire OICC où des traces de neige et des brins de paille constituent l'environnement naturel. Mike est en train d'arranger son motif. L'appareil photo en main, il manipule des objets (des brins de paille) avec lesquels il a constitué déjà une lettre de l'alphabet, la lettre $\ll \mathrm{M} »$.

Le texte que Mike ajoute à son image nous renseigne sur la signification de cette photo et celle de son motif principal. L'auteur nous dit aimer la lettre « $\mathrm{M} »$ qui, en même temps, est la lettre initiale de son prénom. Ensuite, il annonce une autre activité qui suivra l'écriture de ce texte et qui est celle d'assembler un «nuage» d'objets, tous commençant par la lettre initiale « $\mathrm{M}$ ». Ce passage est intéressant puisqu'il cadre l'acte d'écriture au sein du projet «Photovoice» dans une suite d'évènements de littératie. Ceci nous donne une 
idée de l'ancrage spatio-temporel de cet évènement précis. En plus, le choix lexical fait référence à une pratique de littératie et un type de savoir qui pourraient être attribués plutôt à l'espace scolaire et aux pratiques d'apprentissage de l'alphabet à l'école. L'acte d'identifier des lettres et des sons quant à leur positionnement dans un mot fait partie du répertoire habituel d'exercices d'alphabétisation initiale à l'école. C'est cette routine que l'enfant semble vouloir pratiquer ici. Ce geste est significatif puisqu'il montre que l'apprentissage de l'enfant est un processus continu qui s'étend à travers différents espaces sociaux. Le savoir et les routines d'exercice acquis dans un contexte, tel que celui de l'école, sont réemployés dans d'autres contextes, tels que ceux du foyer ou du centre communautaire.

L'autre aspect que l'on peut remarquer est le contexte dans lequel l'activité d'apprentissage est située. Contrairement aux conditions en cadre scolaire où l'apprentissage se fait souvent entre quatre murs, le projet « Photovoice» invite à explorer le monde extérieur. Ceci rend disponible d'autres types de ressources, différentes de celles proposées par l'école qui s'appuie souvent sur des matériaux imprimés ou pré-fabriqués. Ici, l'enfant découvre des matériaux alternatifs qu'il trouve en pleine nature et qu'il transforme en outils de littératie. Cette forme d'exploration est aussi une manière de se connecter à l'espace de la ville et à ses caractéristiques qui sont différentes du monde arctique et qui représentent une expérience nouvelle pour beaucoup d'Inuit émigrés en milieu urbain. En raison du mode de vie dans l'Arctique, les Inuits se sentent proche de l'environnement naturel et sont enclins à le comprendre et à chercher à «lire » ses règles. Cette proximité peut se retrouver dans la métaphore que Mike utilise lorsqu'il propose de construire un «nuage » de mots. Cette proposition pourrait être lue comme l'acte de mettre ensemble des phénomènes naturels et linguistiques appartenant à des systèmes de connaissances d'ordre différent.

C'est encore Louisa (de l'étude de cas I) qui reprend le thème de l'importance de l'environnement lors de l'entrevue rétrospective. Parlant de sujets qu'elle aimerait poursuivre dans une future édition de «Photovoice» prévue pour l'année prochaine elle suggère :

I think people, no matter what, coming here and being part of the Ottawa Inuit Community Centre here, the reality is that what it's like being an urban Inuk I don't know if you could go any broader - you could do like contrasts between nature and urban and how people really like explore the greenery and stuff, that most of us growing up North didn't actually see. [...] um, to the concrete and the big sky-rises, I can really, you know, doing a contrast, but . . but for some people, this is all they've known. So, you know, um, what it's like to have an identity within ...but, um, and one of the things that I saw in both [photo-voice projects] here was this contrast between nature and the urban setting, so that actually might be a theme in itself . . [...] I mean, like, because 
some people are drawn to the fact that there's greenery in Ottawa and other people are drawn to the fact that it's a city [...] and some of the pieces you have as an Inuk kinda fit into that whole piece.

\section{Conclusion}

Dans cet article nous avons discuté de la méthode «Photovoice» et de sa mise en pratique par la communauté Inuit à Ottawa. Notre recherche montre comment cette communauté indigène transnationale s'est approprié cette ressource de littératie en l'adaptant à ses propres besoins et à un contexte socio-culturel spécifique, celui de la migration. Nous avons vu comment cette méthodologie par sa multimodalité a pu stimuler des pratiques de littératie multiples. Incluant la photographie ainsi que des opportunités variées d'interaction et de production langagières, orales et écrites, la méthode propose une série d'activités de littératie amenant à la création d'artefacts complexes. En mettant en valeur le mode visuel qui est privilégié surtout dans des activités initiales, cette méthode répond de façon particulière aux besoins de la communauté en question. Elle met l'accent sur l'exploration de l'environnement auquel les Inuits se sentent attachés de manière très forte. Cette occasion d'explorer l'entourage constitue une opportunité appréciée par les Inuit en milieu urbain afin de «redécouvrir » l'espace de la ville. La méthode «Photovoice» crée une zone d'expérience co-construite qui se prête à la transmission de savoir transfrontaliers et à la formation d'identités Inuit urbaines. Ces processus sont enrichis par le dialogue intergénérationnel qui est stimulé par l'activité. La méthode "Photovoice» utilisée comme une activité de litteratie familiale offre également un contexte où différents types de savoirs peuvent se rencontrer. Ainsi, elle encourage le dialogue entre les savoirs traditionnels relevant de pratiques linguistiques et culturelles dans l'Arctique et d'autres types de savoirs, tels que ceux relevant de l'apprentissage scolaire.

Enfin, nous tenons à souligner la valeur de la méthode «Photovoice» comme outil de recherche au sein des études du courant des New Literacy Studies. La méthode nous a permis de mieux comprendre la situation des Inuits en milieu urbain, qui est très peu connue en dehors de la communauté et qui, jusqu'à ce jour, n'a fait l'objet que de très peu d'études. La méthode «Photovoice» nous a sensibilisé d'abord au positionnement identitaire des Inuits urbains par rapport à l'espace de la ville, mais surtout à l'importance du mode de vie dans l'Arctique dont les Inuits urbains sont séparés et qu'ils cherchent à recréer dans l'espace urbain afin de se « connecter» à cette terre d'origine.

\section{Références}

Baker, T.A. et C.C. Wang. 2006. Photovoice : Use of a participatory action research method to explore the chronic pain experience in older adults. Qualitative Health 
Research, 16, pp. 1405-1413.

Barton, D. 1994. Literacy : An introduction to the ecology of written language. Oxford, Blackwell.

Barton, D., M. Hamilton et R. Ivanč (dir.). 2000. Situated literacies : Reading and writing in context. Londres, Routledge.

Budach, G. 2003. L'alphabétisation d'adultes en Ontario : La valeur du français entre l'identité culturelle et ressource économique. Dans M. Heller et N. Labrie (dir.), Discours et identités : La francité canadienne entre modernité et la mondialisation. Cortil-Wodon, Éditions Modulaires Européennes, pp. 333-363.

Catalani, C. et M. Minkler. 2010. Photovoice : A review of the literature in health and public health. Health Education \& Behavior, 37, pp. 424-451.

Cope, B. et M. Kalantis (dir.). 2000. Multiliteracies : Literacy learning and the design of social futures. Londres, Routlege.

Freire, P. 1973. Education for critical consciousness. New York, Seabury.

Gallo, M.L. 2002. Picture this : Immigrant workers use photography for communication and change. Journal of Workplace Learning, 14(2), pp. 49-57.

Graziano, K.J. 2004. Oppression and resiliency in a post-apartheid South Africa : Unheard of Black gay men and lesbians. Cultural Diversity and Ethnic Minority Psychology 10, pp. 302-313.

Kress, G. 2001. Multimodal teaching and learning : The rhetorics of the science classroom. Londres, Routledge.

Lykes, M.B., M.T. Blanche et B. Hamber. 2003. Narrating survival and change in Guatemala and South Africa : The politics of representation and a liberatory community psychology. American Journal of Community Psychology, 31, pp. 79-90.

Martin-Jones, M. et K. Jones (dir.). 2000. Multilingual literacies : Reading and writing different worlds. Amsterdam, John Benjamins.

Ottawa Inuit Children Centre (OICC). 2009. Nipivut Final Report 2009. Consulté le 29 août 2010. Disponible à : www.ottawainuitchildrens.com/eng/index.php?option=com_ content $\backslash$ \&view $=$ article $\backslash \&$ id $=36 \backslash \& \mid t e m i d=41$.

Strack, R., C. Magill et K. McDonagh. 2004. Engaging youth through Photovoice (Baltimore). Health Promotion Practice, 5, pp. 49-58.

Street, B. 1995. Social literacies : Critical approaches to literacy in development, ethnography and education. Londres, Longman.

Street, B. 2000. Literacy events and literacy practices : Theory and practice in the New Literacy Studies. Dans M. Martin-Jones et K. Jones (dir.), Multilingual literacies : Reading and writing different worlds. Amsterdam, John Benjamins, pp. 17-29.

Tomiak, J. et D. Patrick. 2010. Transnational migration and indegineity in Canada : A case study of urban Inuit. Dans M.C. Forte, Indigenous cosmopolitans : Transnational and transcultural indigineity in the twenty-first century. Bern, Peter Lang, pp. $127-144$.

Wang, C.C. 1999. Photovoice : A participatory action research strategy applied to women's health. Journal of Women's Health, 8, pp. 185-192. 
Wang, C.C. et M. Burris. 1994. Empowerment through photo novella : Portraits of participation. Health Education Quarterly, 21, pp. 171-186.

Wang, C.C. et M. Burris, M. 1997. Photovoice : Concept, methodology, and use for participatory needs assessment. Health Education \& Behavior, 24, pp. 369-387.

Wang, C.C., J.L. Cash et L.S. Powers. 2000. Who knows the streets as well as the homeless? Promoting personal and community action research through Photovoice. Health Promotion Practice 1, pp. 1-89.

Wang, C.C., W.K. Yi, Z.W. Tao et K. Carovano. 1998. Photovoice as a participatory health promotion strategy. Health Promotion International 13, pp. 75-86.

Willson, K., K. Green, M. Haworth-Brockman et R. Rapaport Beck. 2006. Prairie women use Photovoice methods to fight poverty. Canadian Women's Studies, 25, pp. $160-166$.

Wilson, N., S. Dasho, A.C. Martin, N. Wallerstein, C.C. Wang et M. Minkler. 2007. Engaging young adolescents in social action through Photovoice - The Youth Empowerment Strategy (YES !) project. Journal of Early Adolescence, 27, pp. 241-261. 